\title{
Marginal zone lymphoma of the dura - two case reports with long-term follow-up
}

\author{
Felipe de Azevedo Rosas ${ }^{1}$, Sergio Leonardo Favareto ${ }^{1}$, Garles Miller Matias Vieira ${ }^{2}$, \\ Mariana Netto de Oliveira ${ }^{3}$, Felipe D’Almeida Costa ${ }^{4}$, Douglas Guedes de Castro ${ }^{1}$ \\ ${ }^{1}$ Department of Radiation Oncology, AC Camargo Cancer Center, São Paulo, Brazil \\ ${ }^{2}$ Department of Onco-Hematology, AC Camargo Cancer Center, São Paulo, Brazil \\ ${ }^{3}$ Department of Onco-Hematology, Centro Paulista de Oncologia, São Paulo, Brazil \\ ${ }^{4}$ Department of Anatomic Pathology, AC Camargo Cancer Center, São Paulo, Brazil
}

\begin{abstract}
Primary MALT lymphoma arising at the dura is a rare circumstance with no categorical therapeutic plan in literature. There are few reports available with different treatment courses. Here, we report two cases with a long-term follow-up after the same pattern of management and review the literature.
\end{abstract}

Key words: marginal zone B-cell lymphoma; dura-mater; central nervous system neoplasm; MALT lymphoma

Rep Pract Oncol Radiother 2021;26(1):138-142

\section{Introduction}

Marginal zone B-cell lymphomas (MZBCL) are responsible for $7-8 \%$ of all B-cell lymphomas, with a median age of 60 years at diagnosis. Marginal zone B-cell lymphomas, first described as a distinct low-grade lymphoma of mucosa-associated lymphoid tissue (MALT), is frequently associated with the gastric mucosa (30\% of all MALT lymphomas) [1]. Other possible sites widely described in literature are head and neck (30\%), ocular adnexa (24\%), lungs (12\%), skin (12\%), thyroid (7\%) and breasts (2\%) [2].

MALT lymphomas usually present themselves with indolent course and etiology related to lymphoid proliferation due to immune chronic stimulation by infectious agents (ex. Helicobacter Pylori, Campylobacter Jejiuni) or autoimmune diseases (such as Hashimoto tireoiditis, Crohn's disease) [3].

There are few publications about MALT lymphomas of the dura with nearly 100 reported cases in the literature [4]. The lack of prospective studies reflects the inconsistency in therapeutic approach, even among patients treated at the same institution. In this manuscript, we report two patients diagnosed with MALT lymphoma of the dura, both receiving the same treatment at our department and with a long follow-up time (6 and 8 years).

\section{Case reports}

\section{Case 1}

A 59-year-old woman presented a history of one episode of seizure in August, 2010. She denied previous episodes and after screening the central nervous system (CNS) a lesion was observed in cranial magnetic resonance imaging (MRI). The lesion was characterized as an extra-dural and subdural expansive formation with diffuse post contrast enhancement of approximately $1.5 \mathrm{~cm}$ in diameter with vasogenic edema of the adjacent parenchyma. A craniotomy was performed with total resection

Address for correspondence: Sergio Leonardo Favareto, Department of Radiation Oncology, AC Camargo Cancer Center, São Paulo, Brazil, e-mail: leonardo.favareto1@gmail.com

This article is available in open access under Creative Common Attribution-Non-Commercial-No Derivatives 4.0 International (CC BY-NC-ND 4.0) license, allowing to download articles and share them with others as long as they credit the authors and the publisher, but without permission to change them in any way or use them commercially 


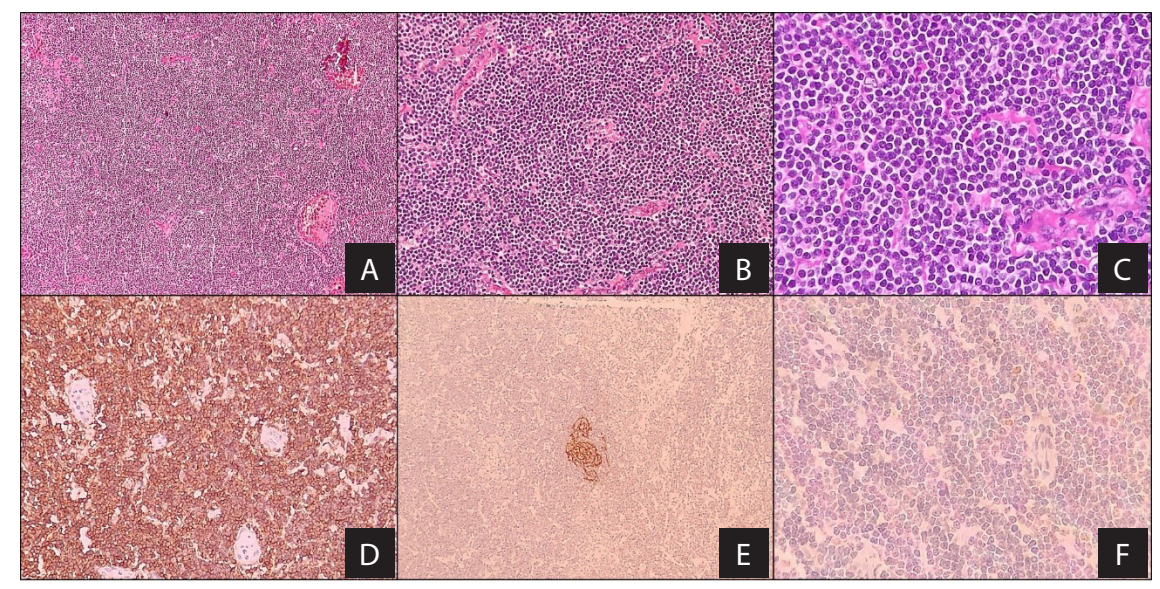

Figure 1. A. Duramater was diffusely infiltrated by sheets of small neoplastic lymphocytes (H\&E, 100X). B. Remnants of colonizes lymphoid follicles could be seen (H\&E, 200X). C. Most of the cells had clear cytoplasm, giving a monocytoid appearance (H\&E, 400X). D. Those cells showed strong and diffuse CD20 staining (CD20, 200X). E. CD21 antibody highlighted the residual meshwork of follicular dendritic cells (CD21, 200x). F. The proliferating index was low, with weak staining in less than $5 \%$ of the cells $(\mathrm{Ki}-67,400 \times)$

of the lesion. Microscopic examination of the lesion showed diffuse dural infiltration by small monotonous lymphocytes, with some regressive and colonized lymphoid follicles. Immunohistochemistry demonstrated positivity for CD20 and Bcl-2, with residual meshwork of follicular dendritic cells highlighted by CD23 and a low proliferative index, consistent with the diagnosis of an extranodal marginal zone lymphoma (Fig. 1). The patient was then referred to our institution where it was decided to deliver adjuvant conformal radiotherapy with a dose of $21 \mathrm{~Gy}$ in 14 fractions to the whole brain and a boost of $9 \mathrm{~Gy}$ in 6 fractions to the tumor bed, completing a total dose of 30 Gy delivered in 20 fractions of 1.5 Gy (Fig. 2). Patient kept follow-up visits every 6 months with cranial MRI during the first year and annually after that, with no evidence of disease recurrence after a follow-up time of 7 years (Fig. 3). After 8 years and 4 months, she had no clinical complaints nor signs of cognitive impairment.

\section{Case 2}

A 62-year-old woman started with seizures in December 2011 and afterwards in September 2012. At the investigation, a cranial MRI showed extra-axial lesions compromising the pachymeningeal coating of a great part of the left cerebral hemisphere, with approximately $2.5 \mathrm{~cm}$ at the maximum width. They were initially suspected to be meningioma based on the MRI and a subtotal resection of the lesions was performed in December, 2012. She was then

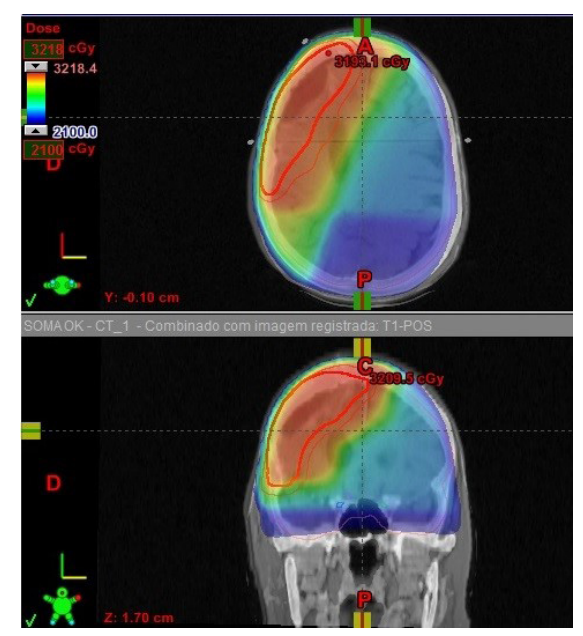

Figure 2. Treatment planning with 3D technique and dose distribution between 2100 and 3218.4 cGy

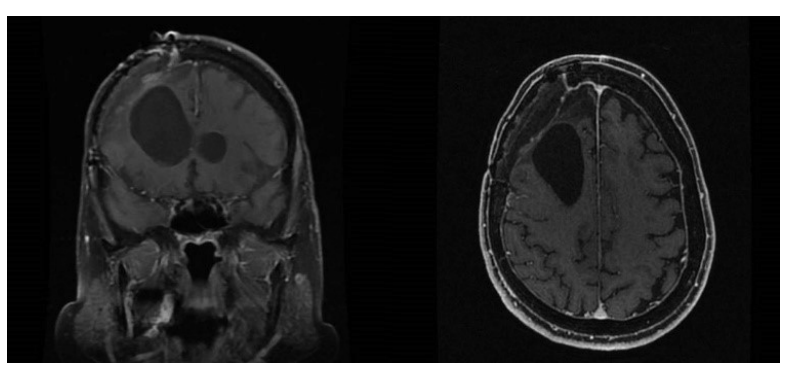

Figure 3. RM of the brain after 5 years of irradiation exhibiting good local control of the disease and no sign of recurrence

referred to our department. The histopathological analysis of the lesions described similar features as 


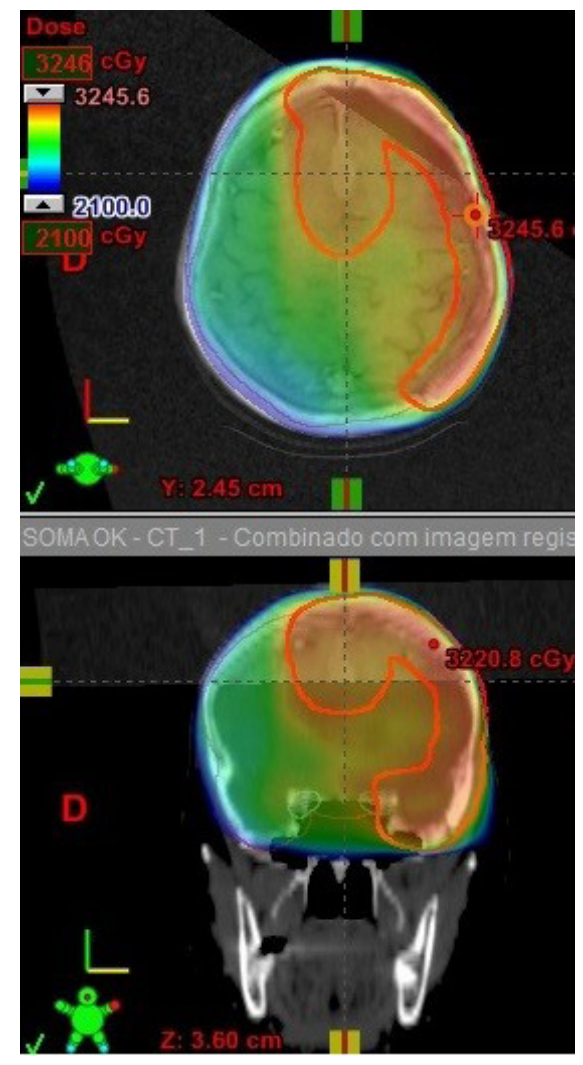

Figure 4. Treatment planning with IMRT technique and dose distribution between 2100 and 3245.6 cGy

those in case one, consistent with marginal zone B-cell lymphoma/MALT. Post-operative cranial MRI revealed significant residual disease. It was decided to deliver adjuvant intensity-modulated radiotherapy (IMRT with a dose of 21 Gy in 14 fractions to the whole brain and a boost of 9 Gy in 6 fractions to the tumor bed, completing a total dose of 30 Gy delivered in 20 fractions of 1.5 Gy (Fig. 4). Patient kept follow-up visits every 6 months with cranial MRI during the first year and annually after that. A complete response was achieved with no signs of disease recurrence, after a follow-up time of 6 years and 9 months (Fig. 5). She also had no clinical complaints nor signs of cognitive impairment at the last follow-up visit.

\section{Discussion}

Primary central nervous system lymphoma (PCNSL) is a non-Hodgkin extranodal lymphoma of the brain, neuro-ocular structures, or spinal cord. Although infiltration into adjacent meninges is occasionally seen, exclusively meningeal involvement is extremely rare. Treatment is based on radio-

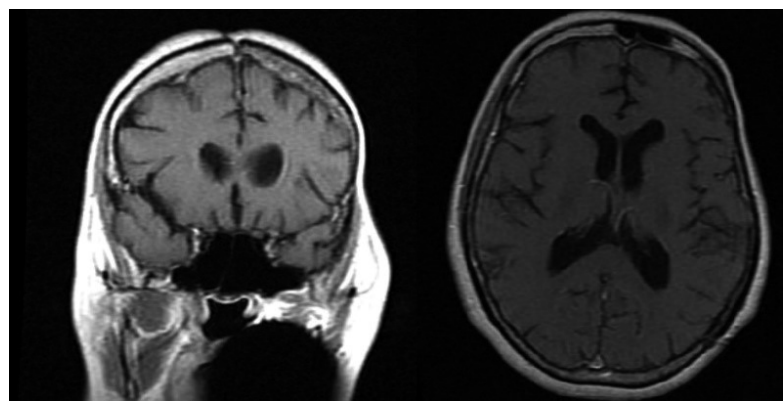

Figure 5. RM of the brain after 3 years of irradiation with good local control of the disease and no sign of recurrence

therapy and intrathecal chemotherapy, frequently involving severe neurotoxicity [5]. Nevertheless, although $90 \%$ of PCNSLs are diffuse large B-cell lymphomas with poor prognosis, there are indolent histological variations with a more favourable clinical course; therefore, accurate recognition of these rare subtypes is of utmost importance. One of these subtypes is lymphoma of mucosa-associated lymphoid tissue (MALT).

When occurring in the CNS, MALT lymphomas generally arise at the dura, in contrast to other histological subtypes that present themselves mainly at the brain parenchyma. It is a rare condition, with no prospective studies and with few published case reports with heterogeneous therapeutic approaches.

There is still no clear explanation for its pathogenesis. One of the most plausible hypotheses is that a sub-clinic inflammation of the dura could recruit lymphocytes to the region from which a monoclonal expansion could originate and, eventually, lymphoma arises. Regarding the role of infectious agents in the development of the disease, none were identified [2]. Due to a strong predominance in the female population, one could hypothesize a hormonal role in its pathophysiology [8].

Initially reported by Kumar et al. [6] in 1997, 15 years after the first MALT lymphomas description, there are nearly 100 reported cases in literature of MALT arising at the dura[4]. Appearing more frequently in the fourth and fifth decades (sooner than most indolent lymphoproliferative neoplasms) [7], with a male to female ratio of $1: 5$, it is usually characterized as a slowly growing dural mass with no infiltrative aspect or systemic involvement. The most frequent symptoms are headaches, dizziness, nausea, vomitting and a slow and progressive memory impairment [9]. 
At CT/MRI scans, MALT lymphomas bear a distinct resemblance to meningiomas, therefore being usually misdiagnosed at first. It presents itself as an extra axial, solid lesion, frequently exhibiting a sign known as "dura tail", which is a contrast enhancing, filiform prolongation of the dura, suggestive but not specific of meningiomas in MRIs. Furthermore, the typical MRI signal intensity characteristics consist of isointensity to slight hypointensity relative to grey matter on the T1-weighted sequence and isointensity to slight hypointensity relative to grey matter on the T2 sequence. Beside that, it demonstrates homogeneous and intense contrast enhancement.

Due to its rarity, there is scarce evidence to guide medical decision. In MALT lymphomas of other sites (not associated with infectious causes), treatment involving radiotherapy or surgical resection offers good results [9]. In a systematic review by Beltran et al. [8] that included 91 patients with primary MALT lymphomas of the dura, $70 \%$ received surgical treatment, $73 \%$ radiotherapy and $37 \%$ chemotherapy. Chemotherapy included intrathecal methotrexate, $\mathrm{CHOP}$ scheme, among others. Because the role of chemotherapy is not entirely clear and commonly associated with severe toxicity, there is a chance that we may be overtreating some patients. Radiotherapy is not considered a standard treatment in patients with this tumor. The radiation volume varied between whole brain radiotherapy (WBRT), involved field radiotherapy and a combination of both. When combined, the mean whole brain dose was $36 \mathrm{~Gy}(21-40 \mathrm{~Gy})$ with boost up to 50 Gy (36-60 Gy) in involved fields.

The recent International Lymphoma Radiation Oncology Group (ILROG) guideline suggests that if more than 1 lesion is present, whole brain to 24 Gy and boosting the involved sites with additional 12 Gy should be indicated. A single lesion may be treated with the presurgical/biopsy MRI volume to the gross tumor Volume plus margins constituting the clinical target volume to 30 to 36 Gy [11]. Suchrecommendation is quite similar to our approach, justified by the diffuse dural involvement.

In the largest published series on dural lymphoma, with 26 patients treated at the Memorial Sloan Kettering Cancer Center and University of Miami, 17 patients underwent resection and 9 had a biopsy. From the patients who received radiotherapy, 13 received focal intensity-modulated radiotherapy (16 to 36 Gy in 9 to 20 fractions) and 6 WBRT
(36 to 39 Gy in 20 to 26 fractions), 3 of whom had multifocal disease. One patient received chemotherapy followed by WBRT ( 45 Gy in 25 fractions). Only 4 patients relapsed (2 local and 2 systemic) after a median follow-up of 64 months. The 3-year progression free survival was $89 \%$ and all patients were alive at last follow-up.[12].

Bustoros et al. [4] reviewed 104 patients in a literature search. Most of them (64\%) were treated with two modalities including resection, RT or chemotherapy, and the majority achieved complete remission on follow-up (only 12 relapses). These data confirmed the favorable prognosis of dural lymphomas and showed a high salvage rate in the case of relapse.

Based on the few case reports available, with limited follow-up time, and the radiosensitivity of this histological subtype, we believe that surgical treatment, especially in cases with mass effect symptoms, followed by radiotherapy alone may offer adequate disease control. Both reported cases were treated with this rationale, achieving satisfactory oncologic and toxicity outcomes after a long-term follow-up (100 and 81 months).

\section{Conflict of interest}

None declared.

\section{Funding}

None declared.

\section{References}

1. Olszewski AJ, Castillo JJ. Survival of patients with marginal zone lymphoma: analysis of the Surveillance, Epidemiology, and End Results database. Cancer. 2013; 119(3): 629-638, doi: 10.1002/cncr.27773, indexed in Pubmed: 22893605.

2. Zucca E, Conconi A, Roggero E. Non-gastric MALT lymphomas: a survey of 369 European patients. The International Extranodal Lymphoma Study Group. Ann Oncol. 2000; 11(Suppl 4): 1-2, doi: Abstr. 444.

3. Zucca E, Bertoni F. The spectrum of MALT lymphoma at different sites: biological and therapeutic relevance. Blood. 2016; 127(17): 2082-2092, doi: 10.1182/ blood-2015-12-624304, indexed in Pubmed: 26989205.

4. Bustoros M, Liechty B, Zagzag D, et al. A Rare Case of Composite Dural Extranodal Marginal Zone Lymphoma and Chronic Lymphocytic Leukemia/Small Lymphocytic Lymphoma. Front Neurol. 2018; 9: 267, doi: 10.3389/ fneur.2018.00267, indexed in Pubmed: 29740389.

5. Schaff LR. Updates on Primary Central Nervous System Lymphoma. Curr Oncol Rep. 2018; 20(2): 11, doi: 10.1007/ s11912-018-0666-1, indexed in Pubmed: 29492682. 
6. Kumar S, Kumar D, Kaldjian EP, et al. Primary low-grade B-cell lymphoma of the dura: a mucosa associated lymphoid tissue-type lymphoma. Am J Surg Pathol. 1997; 21(1): 81-87, doi: 10.1097/00000478-199701000-00009, indexed in Pubmed: 8990144.

7. A Clinical Evaluation of the International Lymphoma Study Group Classification of Non-Hodgkin's Lymphoma. Blood. 1997; 89(11): 3909-3918, doi: 10.1182/blood.v89.11.3909, indexed in Pubmed: 9166827.

8. Beltrán BE, Kuritzky B, Quiñones P, et al. Extranodal marginal zone lymphoma of the cranial dura mater: report of three cases and systematic review of the literature. Leuk Lymphoma. 2013; 54(10): 2306-2309, doi: 10.3109/1042 8194.2013.771399, indexed in Pubmed: 23363270.

9. Kamoshima Y, Sawamura Y, Sugiyama T, et al. Primary central nervous system mucosa-associated lymphoid tissue lymphoma - case report. Neurol Med Chir (Tokyo).
2011; 51(7): 527-530, doi: 10.2176/nmc.51.527, indexed in Pubmed: 21785250.

10. Thieblemont C, Zucca E. Clinical aspects and therapy of gastrointestinal MALT lymphoma. Best Pract Res Clin Haematol. 2017; 30(1-2): 109-117, doi: 10.1016/j. beha.2017.01.002, indexed in Pubmed: 28288705.

11. Yalahom J, Illidge T, Specht $L$, et al. Modern radiation therapy for extranodal lymphomas: field and dose guidelines from the ernational Lymphoma Radiation Oncology Group. Int J RadiationOncology Biol Phys. 2015; 92(1): 11-315, doi: 10.1016/j.jirobp.2015.01.009, indexed in Pubmed: 25863750.

12. de la Fuente MI, Haggiagi A, Moul A, et al. Marginal zone dural lymphoma: the Memorial Sloan Kettering Cancer Center and University of Miami experiences. Leuk Lymphoma. 2017; 58(4): 882-888, doi: 10.1080/10428194.20 16.1218006, indexed in Pubmed: 27649904. 